McGill Law Journal

Revue de droit de McGill

\title{
De Dunsmuir à Khosa
}

\section{Louis LeBel}

Volume 55, numéro 2, july 2010

URI : https://id.erudit.org/iderudit/045087ar

DOI : https://doi.org/10.7202/045087ar

Aller au sommaire du numéro

Éditeur(s)

McGill Law Journal / Revue de droit de McGill

ISSN

0024-9041 (imprimé)

1920-6356 (numérique)

Découvrir la revue

Citer cet article

LeBel, L. (2010). De Dunsmuir à Khosa. McGill Law Journal / Revue de droit de McGill, 55(2), 311-327. https://doi.org/10.7202/045087ar
Résumé de l'article

L'exercice du contrôle judiciaire en droit administratif canadien a récemment été réexaminé par la Cour suprême du Canada dans le cadre des arrêts Dunsmuir c. Nouveau Brunswick et Canada (Ministère de la Citoyenneté et de l'Immigration) c. Khosa. L'auteur se penche sur l'impact de ces deux décisions à la lumière des raisons qui ont poussé la Cour à modifier certains aspects fondamentaux du contrôle judiciaire.

L'auteur évalue d'abord l'impact de l'arrêt Dunsmuir par rapport à l'équité procédurale, un aspect souvent négligé de cette décision de la Cour. L’auteur présente ensuite un commentaire sur la réforme des normes de contrôle judiciaire introduite par ce même arrêt, et ce, à travers le concept de déférence et son rôle dans ce domaine du droit administratif. Finalement, l'auteur aborde l'arrêt Khosa pour analyser la relation entre l'approche des normes de contrôle développée dans Dunsmuir et celle découlant des régimes législatifs de contrôle judiciaire. Une telle analyse permet de faire le point sur l'évolution récente dans ce domaine complexe du droit administratif canadien.
Ce document est protégé par la loi sur le droit d'auteur. L'utilisation des services d’Érudit (y compris la reproduction) est assujettie à sa politique d'utilisation que vous pouvez consulter en ligne.

https://apropos.erudit.org/fr/usagers/politique-dutilisation/ 
McGill Law Journal Revue de droit de McGill

\section{DE DUNSMUIR À KHOSA}

\section{L'honorable Louis LeBel*}

L'exercice du contrôle judiciaire en droit administratif canadien a récemment été réexaminé par la Cour suprême du Canada dans le cadre des arrêts Dunsmuir c. Nouveau Brunswick et Canada (Ministère de la Citoyenneté et de l'Immigration) c. Khosa. L'auteur se penche sur l'impact de ces deux décisions à la lumière des raisons qui ont poussé la Cour à modifier certains aspects fondamentaux du contrôle judiciaire.

L'auteur évalue d'abord l'impact de l'arrêt Dunsmuir par rapport à l'équité procédurale, un aspect souvent négligé de cette décision de la

Cour. L'auteur présente ensuite un commentaire sur la réforme des normes de contrôle judiciaire introduite par ce même arrêt, et ce, à travers le concept de déférence et son rôle dans ce domaine du droit administratif. Finalement, l'auteur aborde l'arrêt Khosa pour analyser la relation entre l'approche des normes de contrôle développée dans Dunsmuir et celle découlant des régimes législatifs de contrôle judiciaire. Une telle analyse permet de faire le point sur l'évolution récente dans ce domaine complexe du droit administratif canadien.$$
\text { du droit administratif canadien. }
$$

In Dunsmuir v. New Brunswick and Canada (Minister of Citizenship and Immigration) $v$. Khosa, the Supreme Court of Canada recently re-examined the exercise of judicial review in Canadian administrative law. The author evaluates the impact of these two decisions in light of the motivations that led the Court to modify certain fundamental elements of judicial review.

The author begins by assessing the impact of the Dunsmuir decision on procedural fairness, an often ignored aspect of the decision. The author then presents Dunsmuir's discussion regarding the standard-of-review reform through the concept of deference and its role in administrative law. Finally, the Khosa decision is used to present an analysis of the relationship between the standard of review approach introduced in Dunsmuir, and the standard of review defined in legislative frameworks of judicial review. Such an inquiry allows the author to outline recent developments and to clarify the current state of the law in this complex domain of Canadian administrative law.

* Juge à la Cour suprême du Canada. Ce texte est une version remaniée d'une allocution prononcée le 7 octobre 2009, lors d'une conférence organisée par l'Association du Jeune Barreau de Montréal, et le 11 mars 2010, devant les juges de la Cour supérieure du Québec et de la Cour du Québec.

(C) Louis LeBel 2010

Citation: (2010) 55 McGill L.J. $311 \sim$ Référence : (2010) 55 R.D. McGill 311 
312 (2010) 55 MCGILl LAW JOURNAL REVUE DE DROIT DE MCGILL

$\begin{array}{ll}\text { Introduction } & 313\end{array}$

I. Dunsmuir et l'équité procédurale 313

II. Dunsmuir et les normes de contrôle judiciaire 318

A. Révision des normes de contrôle 319

B. Révision de la méthode pragmatique et fonctionnelle 322

III. L'arrêt Khosa 325

$\begin{array}{ll}\text { Conclusion } & 327\end{array}$ 


\section{Introduction}

L'examen de la jurisprudence récente sur le contrôle judiciaire en droit administratif au Canada rend nécessaire une réflexion attentive sur certains principes fondamentaux du droit canadien. Par exemple, le concept de déférence, qui joue un rôle si fondamental en droit administratif, implique des aspects de la primauté du droit, de la séparation constitutionnelle des pouvoirs et de la suprématie législative. L'application de ces principes fondamentaux n'a cependant pas permis de développer aisément un cadre cohérent pour l'exercice de la fonction de contrôle judiciaire, comme en témoigne l'histoire de celui-ci.

L'arrêt récent de la Cour suprême du Canada dans Dunsmuir c. Nouveau-Brunswick ${ }^{1}$, rendu en mars 2008, ainsi que l'arrêt Canada (Ministre de la Citoyenneté et de l'Immigration) c. Khosa ${ }^{2}$, prononcé en mars 2009, ont examiné les complexités du contrôle judiciaire au Canada. J'entends aborder la manière dont ces arrêts ont modifié les perspectives du contrôle judiciaire.

Je m'attaquerai d'abord à l'étude de l'arrêt Dunsmuir. Cette décision a permis à la Cour de réexaminer l'analyse de contrôle judiciaire et de reconsidérer les exigences de l'équité procédurale à l'égard de la cessation de l'emploi des titulaires de charges publiques. Puisque cet aspect de Dunsmuir est souvent négligé, je commencerai mon analyse en rappelant l'impact de cet arrêt sur les titulaires de charges publiques et sur les principes de justice naturelle qui avaient été énoncés dans l'arrêt Knight $c$. Indian Head School Division No. 1933 en 1990.

\section{Dunsmuir et l'équité procédurale}

Avant l'arrêt Dunsmuir, les tribunaux maintenaient une distinction nette entre les employés publics liés par un contrat régulier d'emploi et ceux qui étaient titulaires d'une charge publique. Seuls ces derniers avaient droit à l'exécution d'obligations d'équité procédurale en cas de renvoi. De plus, les jurisprudences canadienne et britannique avaient établi une distinction encore plus étroite entre les titulaires de charges publiques qui pouvaient être renvoyés seulement pour motif et ceux qui servaient la Couronne à son bon plaisir. On reconnaissait aux premiers des droits à l'équité procédurale dans le cas de cessation de leur fonction. Au-

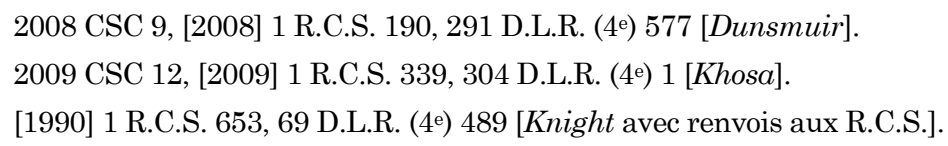


cune obligation d'équité n'était due aux seconds ${ }^{4}$. La distinction problématique entre ces deux types de titulaires de charges publiques a été rejetée dans Knight. Dans ce jugement, la majorité de la Cour a décidé que le directeur des services éducatifs dans une réserve indienne, qui occupait une fonction au bon plaisir de son employeur, avait quand même droit à l'exécution d'obligations d'équité procédurale avant son renvoi ${ }^{5}$. La Cour a constaté la difficulté de classer les titulaires de charges publiques dans ces catégories précises. Elle a aussi noté l'importance de s'assurer que les organismes administratifs exercent correctement leurs pouvoirs délégués et ce, même à l'égard des employés qu'ils engagent selon leur bon plaisir. Ces facteurs ont amené la Cour à reconnaître que des titulaires de charges publiques, comme M. Knight, ont droit à des protections procédurales minimales, comme le droit d'être entendus, avant leur renvoi. Cependant, malgré l'abolition d'une partie de ces distinctions problématiques, la Cour a continué à faire la distinction entre le statut des titulaires d'une charge publique et celui d'employés liés par contrat, qui se situaient dans une relation de "maître et serviteur" avec la Couronne, selon le langage un peu désuet qu'employait Lord Reid dans l'arrêt fondateur Ridge v. Baldwin ${ }^{6}$.

Dans l'arrêt Knight, la Cour décida que l'administration scolaire en cause était tenue à des obligations substantielles de justice naturelle à l'égard de l'employé en raison du caractère final de la décision et de son impact sur la vie professionnelle de l'employé et sur sa capacité à gagner sa vie. Pour exécuter son obligation, l'employeur devait alors communiquer à l'employé les motifs de son renvoi et lui donner une occasion de s'expliquer avant de procéder à son congédiement. En l'espèce, l'administration scolaire avait respecté ces deux obligations ${ }^{7}$. Le juge Sopinka, écrivant au nom de la minorité, n'avait pas voulu reconnaître l'existence d'une obligation d'équité naturelle, mais avait exprimé son accord avec la conclusion selon laquelle l'employé avait bénéficié de toutes les garanties procédurales pertinentes.

Dans l'arrêt Dunsmuir, la Cour a en partie confirmé, mais aussi partiellement modifié, l'approche qu'elle avait adoptée dans Knight. Tout d'abord, la Cour a confirmé certains éléments de la décision Knight au sujet de l'existence des obligations générales d'équité ou de justice naturelle auxquelles sont tenus les décideurs administratifs ${ }^{8}$. Tous les membres de

4 Voir Ridge v. Baldwin (1963), [1964] A.C. 40, [1963] 2 All E.R. 66 (H.L.) ; Nicholson c. Haldimand-Norfolk Regional Police Commissionners, [1979] 1 R.C.S. 311, 88 D.L.R. (3e) 671.

$5 \quad$ Knight, supra note 3 aux pp. 675-76.

6 Supra note 4.

$7 \quad$ Knight, supra note 3 aux pp. 684-86.

8 Dunsmuir, supra note 1 au para. 81. 
la Cour ont cependant abandonné la conception voulant que tous les titulaires d'une charge publique aient droit à l'exécution d'une obligation d'équité procédurale ou de justice fondamentale dans les questions relatives à leurs renvois, mais que cette obligation ne s'applique pas aux employés publics protégés par un contrat d'emploi. Si un contrat existe, le rapport d'emploi demeure gouverné par le droit du contrat plutôt que par des principes de justice naturelle, et ce, même si l'employé se trouve aussi titulaire d'une charge publique.

L'arrêt Dunsmuir abolit ainsi la distinction entre un titulaire d'une charge publique et un employé lié par un contrat ordinaire. Il pousse encore plus loin la simplification de l'analyse du statut juridique de l'employé public qui avait commencé dans l'arrêt Knight ${ }^{9}$. Comme je l'ai écrit avec le juge Bastarache, cette distinction avait été difficile à maintenir. Elle laissait dans des zones grises et incertaines des groupes d'employés qu'il était difficile de catégoriser, tels que les médecins rattachés au Service de santé publique ou les administrateurs de niveau intermédiaire dans les municipalités ${ }^{10}$.

Cependant, les difficultés relatives à la catégorisation des employés liés par contrat et des titulaires de charges publiques n'étaient pas seules en cause. Des motifs de principe et des raisons d'ordre pratique justifiaient cette réévaluation de l'arrêt Knight. La nature même des règles de justice naturelle était en cause. En effet, celles-ci visent au moins en partie à protéger les intéressés contre l'exercice arbitraire et illégitime de l'autorité des pouvoirs délégués par la loi. Dans les cas où un employeur renvoie son employé de bonne foi, conformément aux dispositions d'un contrat d'emploi, on conçoit difficilement comment cette préoccupation peut être pertinente. Un renvoi avec un préavis raisonnable ou pour un motif suffisant ne paraît pas en soi injuste ${ }^{11}$.

D'un point de vue plus pratique, les recours d'un employé renvoyé en droit commun, qu'il s'agisse de la common law ou du droit civil, peuvent en réalité être plus efficaces que ceux que reconnaît le droit administratif. En common law et en droit civil, par exemple, la période de préavis tient compte de facteurs divers tels que l'âge, la durée du service, l'expérience et la possibilité de retrouver un emploi analogue. Un employé public pourrait aussi obtenir des dommages additionnels dans le cas où son renvoi aurait été motivé par la mauvaise foi, ainsi que s'il reçoit ou bénéficie des

$9 \quad$ Ibid. aux para. 79-117.

$10 \quad$ Ibid. au para. 94.

11 Ibid. au para. 105. 
protections additionnelles des normes du travail ou des législations sur les droits de la personne ${ }^{12}$.

À l'inverse, les recours administratifs peuvent parfois entraîner des résultats injustes pour l'employeur ou pour l'employé. Ainsi, une violation de l'équité fondamentale rend la décision de renvoi nulle dès l'origine. L'employé rétablit alors la situation qui existait avant son renvoi et reçoit son salaire et ses indemnités pour le temps écoulé entre la décision de l'employeur et le jugement. L'employeur peut cependant renvoyer l'employé de nouveau, en respectant cette fois les exigences procédurales imposées par les principes de justice fondamentale. D'autres considérations, comme la possibilité pour l'employé de se trouver un autre emploi, deviennent à ce moment étrangères à l'analyse. Par ailleurs, l'employeur ne saurait invoquer l'obligation de l'employé de limiter le préjudice puisque des salaires impayés ne s'assimilent pas aux dommages-intérêts. De cette façon, il pourrait être exposé à payer des traitements qui n'auraient pas dû normalement être versés, selon la durée des procédures judiciaires ${ }^{13}$.

La Cour suprême du Canada était fort consciente de ces questions avant même l'arrêt Dunsmuir. Ainsi, dans Wells c. Terre-Neuve ${ }^{14}$, le titulaire d'un emploi de la fonction publique avait été renvoyé lorsqu'une loi avait aboli son poste. Malgré le caractère législatif ou statutaire de sa relation d'emploi, la Cour avait alors décidé que l'employé avait droit d'exercer les recours de common law pour violation de contrat à la suite de son renvoi. Le juge Major, écrivant pour la Cour, avait alors souligné, fort à propos, les aspects contractuels importants de la relation juridique entre le fonctionnaire et l'État :

Un examen fondé sur le bon sens de ce que signifie le fait de travailler pour le gouvernement tend à indiquer que ces relations portent toutes les marques d'un contrat. Des négociations donnent lieu à une entente et à un emploi et engendrent des obligations exécutoires pour les deux parties. La Couronne agit en grande partie comme un citoyen ordinaire le ferait, s'engageant dans des relations commerciales avantageuses pour les deux parties, tant avec des particuliers qu'avec des sociétés. Bien que la Couronne puisse être tenue de suivre des lignes directrices prévues par la loi, le résultat demeure quand même un contrat de travail ${ }^{15}$.

12 Ibid. aux para. 108, 111. Pour un jugement récent sur l'évaluation des dommages dans le cas d'un renvoi de mauvaise foi, voir Honda Canada c. Keays, 2008 CSC 39, [2008] 2 R.C.S. 362 aux para. 87-90, 294 D.L.R. (4e) 577, juge LeBel, dissident.

13 Dunsmuir, supra note 1 aux para. 108-109.

14 [1999] 3 R.C.S. 199, 177 D.L.R. (4e) 73 [Wells avec renvois aux R.C.S.].

$15 \quad$ Ibid. au para. 22. 
De même, certains auteurs ont exprimé des réserves à l'égard des tentatives de catégorisation stricte des employés publics comme titulaires de charges publiques ou comme employés à contrat. Ils ont d'ailleurs souligné l'état incertain et insatisfaisant de la jurisprudence qui s'était développée dans ce domaine ${ }^{16}$.

Les conclusions de l'arrêt Dunsmuir sur cette question de justice naturelle ne signifient toutefois pas qu'un employé public ne pourrait jamais exercer des recours ou des droits provenant du droit administratif lorsque son employeur met fin à son emploi. En l'absence d'un contrat d'emploi, un employé public pourrait toujours invoquer les principes d'équité procédurale pour tenter de se protéger contre un congédiement injuste. De plus, les titulaires de fonctions de l'État régies par la Constitution ${ }^{17}$, comme les juges, ont droit au respect de l'obligation d'équité naturelle puisque la Constitution elle-même, et non pas un simple contrat, détermine les éléments essentiels de leurs rapports juridiques avec l'État ${ }^{18}$. De plus, Dunsmuir reconnaît toujours des droits à l'équité procédurale dans le cas d'employés publics qui remplissent réellement leurs fonctions au bon plaisir de la Couronne, en ce sens qu'on peut les renvoyer sommairement ${ }^{19}$. Enfin, les dispositions mêmes d'une loi qui gouverne une relation d'emploi peuvent établir de telles obligations. Ainsi, lorsqu'une loi accorde certains droits procéduraux, il serait justifié de placer ces droits dans leur contexte pour reconnaître implicitement à leur titulaire certaines garanties procédurales qui s'y rattachent habituellement ${ }^{20}$.

En rendant l'arrêt Dunsmuir, la Cour suprême du Canada n'a jamais voulu réduire l'importance des principes de justice fondamentale dans le droit administratif canadien. Comme je l'ai souligné avec le juge Bastarache, l'équité procédurale demeure, pour la Cour, une pierre angulaire du droit administratif canadien moderne ${ }^{21}$. D'ailleurs, l'exigence d'équité procédurale demeure particulièrement importante dans le contexte des relations d'emploi. Les remarques du juge Dickson, selon lesquelles un niveau

16 Voir David J. Mullan, Administrative Law, Toronto, Irwin Law, 2001 aux pp. 166-67 ; Phyllis Norrie, "Status and Contracts of Employment in the Field of Public Education in Saskatchewan " (1991) 55 Sask. L. Rev. 365.

17 Loi constitutionnelle de 1867 (R.-U.), 30 \& 31 Vict., c. 3, reproduite dans L.R.C. 1985, app. II, n 5 [Constitution].

18 Dunsmuir, supra note 1 au para. 115. Voir aussi Wells, supra note 14 au para. 31.

19 Dunsmuir, supra note 1 au para. 115.

20 Ibid. au para. 116. Dans Malloch v. Aberdeen Corp. ([1971] 1 W.L.R. 1578, [1971] 2 All E.R. 1278 (H.L.)), la House of Lords décida que l'obligation légale de donner un avis de trois semaines d'une proposition de renvoi était réputée inclure un droit de faire des représentations à l'assemblée à laquelle serait discutée la proposition.

21 Dunsmuir, supra note 1 au para. 79. 
élevé de justice procédurale est exigé dans le cas des décisions qui impliquent le droit de continuer à exercer une profession, demeurent tout aussi pertinentes qu'au moment de leur rédaction il y a trente ans ${ }^{22}$. À cet effet, Dunsmuir a tout simplement reconnu que ce niveau élevé de justice naturelle peut être atteint sans nécessairement recourir aux principes de droit administratif. La justice procédurale ne peut être considérée comme figée dans un contexte statique, incapable d'évoluer. En réévaluant et en redéfinissant les situations dans lesquelles ces obligations d'équité procédurale s'appliquent, notre Cour a reconnu la nature évolutive de notre société et a voulu que la justice naturelle se développe en accord avec les normes et valeurs modernes de cette dernière. La mise de côté par Dunsmuir de l'exigence que les titulaires de charges publiques soient nécessairement titulaires d'un droit à l'équité procédurale en cas de renvoi respecte la nature juridique réelle des emplois publics et témoigne de la capacité d'adaptation du droit administratif canadien.

\section{Dunsmuir et les normes de contrôle judiciaire}

Cette contribution importante de l'arrêt Dunsmuir au développement de l'équité procédurale et des principes de justice fondamentale a certainement été occultée par la réévaluation des trois normes traditionnelles de contrôle judiciaire. L'intérêt exprimé dans la communauté juridique à propos de cette décision se comprend bien. Dunsmuir a apporté deux changements importants au droit administratif dans ce domaine. Il a d'abord aboli les deux normes du manifestement déraisonnable et du raisonnable simpliciter pour y substituer une norme unique et unifiée de rationalité. Ensuite, il a modifié la méthode dite " pragmatique et fonctionnelle" pour en faire une méthode plus simple et plus holistique d'analyse de contrôle judiciaire. Cette méthode doit être utilisée pour déterminer le niveau de déférence approprié à l'égard d'un décideur administratif.

Certes, il est encore tôt pour évaluer l'impact de ces changements. Celui-ci sera déterminé avec le développement de la jurisprudence dans les cours de justice et les tribunaux administratifs. Ces changements ont été faits dans l'espoir qu'au terme de cette évolution, se mette en place un système plus efficace de contrôle judiciaire. L'avenir nous indiquera si cet espoir était réaliste.

22 Kane c. Conseil d'administration de l'Université de la Colombie-Britannique, [1980] 1 R.C.S. 1105 à la p. 1113, 110 D.L.R. (3e) 311. 


\section{A. Révision des normes de contrôle}

Le premier changement effectué par la Cour dans Dunsmuir a été de fusionner les deux variations de la norme du déraisonnable en un standard unique. Les critiques du maintien de la distinction entre les deux normes de raisonnabilité sont bien connues. Je m'étais exprimé sur ce sujet dans mes motifs concurrents dans l'affaire de Toronto (Ville de) c. S.C.F.P., section locale $79^{23}$. J'avais alors souligné que la norme du manifestement déraisonnable était au fond trop semblable à celle du raisonnable simpliciter. La distinction dépendait de paramètres vagues et inconsistants qui affectaient sa mise en œuvre. Quel niveau d'examen était approprié pour décider quand une décision pouvait être qualifiée de manifestement déraisonnable ? Quel degré d'irrationalité une décision devait-elle atteindre pour justifier une intervention judiciaire ${ }^{24}$ ?

Les problèmes de la mise en œuvre d'un système fondé sur des degrés d'irrationalité étaient bien illustrés dans certaines remarques du professeur David Mullan : "Like "uniqueness", irrationality either exists or does not. There cannot be shades of irrationality. In other words, I defy any judge or lawyer to provide a concrete example of the difference between the merely irrational and the clearly irrational! ${ }^{25}$.

Malgré tous les efforts des tribunaux dans des causes importantes comme Barreau du Nouveau-Brunswick c. Ryan ${ }^{26}$, ce défi s'avérait à peu près impossible à relever. Les juristes, qu'ils soient juges ou avocats, rencontraient de grandes difficultés lorsqu'ils tentaient de déterminer les bases conceptuelles des deux normes de rationalité. Ainsi, des remarques caustiques du juge Berger, de la Cour d'appel de l'Alberta, avaient souligné les difficultés résultant de la distinction entre le simplement et le manifestement déraisonnable. Il les qualifiait de "twin obstacles of reasonableness and patent unreasonableness which challenge the judicial mind to, among other questionable nuances, distinguish between "probing" and "somewhat probing" analyses ${ }^{27}$. Ces réserves et ces difficultés ont amené la Cour suprême du Canada à réévaluer l'usage des trois normes

232003 CSC 63, [2003] 3 R.C.S. 77, 232 D.L.R. (4e) 385 [Toronto c. S.C.F.P.]. Voir aussi Voice Construction Ltd. c. Construction \& General Workers' Union, Local 92, 2004 CSC 23, [2004] 1 R.C.S. 609 aux para. 39-41, 238 D.L.R. (4e) 217.

24 Toronto c. S.C.F.P., supra note 23 aux para. 101-34

25 David J. Mullan, " Recent Developments in Standard of Review " dans Taking the Tribunal to Court: A Practical Guide for Administrative Law Practitioners, Ontario, Canadian Bar Association, 2000 à la p. 25, tel que cité dans Toronto c. S.C.F.P., supra note 23 au para. 105.

262003 CSC 20, [2003] 1 R.C.S. 247, 223 D.L.R. (4e) 577.

27 Chauvet v. Alberta (Workers' Compensation Board, Appeals Commission), 2007 ABCA 155, 409 A.R. 17 au para. 17, [2007] 8 W.W.R. 649. 
de contrôle et à introduire une nouvelle norme unique qui s'appliquerait en plus, évidemment, de la norme d'exactitude ou de correction.

À cette étape, il est nécessaire de soulever une question apparemment simple, mais en réalité difficile : qu'est-ce que le raisonnable ? Il s'agit d'une question délicate et fondamentale. Des volumes de science juridique lui ont été consacrés dans à peu près tous les domaines du droit, puisqu'on la retrouve dans bien d'autres domaines que le droit administratif. Dans le cas du contrôle judiciaire, le concept de raisonnable se rattache primordialement à la notion de déférence. J'ai souligné dans une conférence antérieure que le concept de déférence demeure mal compris en droit administratif ${ }^{28}$. Pour les fins de mon propos, je tenterai de définir la déférence comme une attitude de respect pour l'intention du législateur et pour la capacité de décision des tribunaux et des décideurs administratifs. Ce concept exige qu'une cour chargée d'exercer la fonction de contrôle judiciaire démontre une attitude de respect authentique pour les décisions des législatures, pour leurs choix législatifs de déléguer des pouvoirs à des corps et tribunaux administratifs. De plus, cette notion exige que les cours fassent preuve d'une certaine sensibilité à l'égard des exigences de l'action des tribunaux administratifs et sachent prendre en compte le contexte particulier dans lequel ces organismes exercent leurs fonctions ${ }^{29}$.

Cependant, cette exigence de déférence ne signifie pas que la cour qui procède à un contrôle judiciaire sur la base de la norme du raisonnable doive identifier un degré particulier de déférence ${ }^{30}$. En réalité, lorsqu'une cour décide que la norme du raisonnable s'applique, elle doit alors décider si le résultat se situe bien dans un ensemble de résultats possibles et acceptables, défendables au regard des faits et du droit ${ }^{31}$. Avec le juge Bastarache, j'ai souligné l'importance, à cet égard, des principes de justification, de transparence et d'intelligibilité pour décrire la norme de contrôle des procédures et des décisions du décideur administratif : est-il possible de justifier la procédure ou le résultat au regard des faits et du droit relatif à la question ? La procédure de décision est-elle demeurée libre de toute influence de facteurs externes qui n'ont pas été clairement articulés ou déclarés ? Le résultat est-il compréhensible en tenant compte du contexte factuel et juridique dans lequel il est survenu ? La déférence, tout comme le raisonnable, ne se prête pas aisément à la quantification.

28 Voir Louis LeBel, « Some Properly Deferential Thoughts on Deference » (2008) 21 Can. J. Admin. L. \& Prac. 1.

29 Ibid. aux pp. 6-7.

30 Cette question a été soulevée par le professeur Mullan. Voir David Mullan, « Dunsmuir v. New Brunswick, Standard of Review and Procedural Fairness for Public Servants: Let's Try Again! » (2008) 21 Can. J. Admin. L. \& Prac. 117 à la p. 135.

31 Dunsmuir, supra note 1 au para. 47. 
Si une attitude de déférence est justifiée, la cour chargée de la révision judiciaire doit examiner la matière soumise à son contrôle, en gardant à l'esprit les idéaux de justification de transparence et d'intelligibilité, mais en acceptant de s'incliner devant une décision rendue dans le respect de ces idéaux. Le concept de déférence implique aussi la nécessité de reconnaître qu'une question peut souvent recevoir plus d'une réponse et que la cour exerçant le pouvoir de contrôle devrait respecter un résultat et un processus décisionnel qui se situent dans le domaine du raisonnable. La cour de révision ne devrait pas chercher à imposer sa propre conception de ce que serait nécessairement une meilleure solution lorsqu'une attitude de déférence s'impose à l'égard d'une décision.

Par contre, si aucune déférence ne se justifie, un contrôle suivant la norme de correction ou d'exactitude s'appliquera. À cet égard, Dunsmuir ne change rien à la nature de la norme de la décision correcte. En vertu de celle-ci, la cour peut substituer sa propre opinion de la décision appropriée à celle qu'avait adoptée le décideur administratif ${ }^{32}$.

Les deux principes qui guident le contrôle judiciaire, c'est-à-dire le respect de la règle de droit et la suprématie législative, sont implicites dans la définition du concept de déférence. Comme je l'ai écrit avec le juge Bastarache dans Dunsmuir, " la primauté du droit est assurée par le dernier mot qu'ont les cours de justice en matière de compétence, et la suprématie législative, par la détermination de la norme de contrôle applicable en fonction de l'intention du législateur $»^{33}$. Dans l'exercice de leurs fonctions de révision des décisions administratives, les tribunaux doivent chercher à réaliser un équilibre approprié entre la nécessité d'assurer la primauté de la règle de droit et celle de reconnaître qu'il n'est pas légitime pour les cours de justice d'interférer avec les décisions des élus et celles des organismes administratifs œuvrant à l'intérieur de leur autorité statutaire. L'adoption d'une attitude appropriée de déférence, dans les cas appropriés, permet aux tribunaux de trouver l'équilibre nécessaire entre ces deux principes concurrents ${ }^{34}$.

L'arrêt Dunsmuir affecte cette recherche d'équilibre de plusieurs façons. D'abord, ce jugement réaffirme la primauté du droit lorsqu'il rejette la norme du manifestement déraisonnable. En effet, celle-ci légitimait des décisions déraisonnables, mais qui ne l'étaient pas suffisamment pour justifier autrement l'intervention ${ }^{35}$. L'arrêt souhaite plutôt que les tribunaux remplissent pleinement le rôle constitutionnel de protection contre

\footnotetext{
$32 \quad$ Ibid. au para. 50.

33 Ibid. au para. 30.

34 Voir LeBel, supra note 28 aux para. 3-4.

35 Dunsmuir, supra note 1 au para. 42.
} 
l'exercice illégal et arbitraire du pouvoir statutaire. En même temps, il insiste sur le respect de la suprématie du législateur en réaffirmant l'importance de la déférence dans l'exercice du contrôle judiciaire de l'action administrative. En effet, le concept de déférence exige la reconnaissance du fait que les tribunaux et les décideurs administratifs jouent des rôles fort différents à l'intérieur du système constitutionnel canadien ${ }^{36}$. De cette manière, Dunsmuir maintient le principe de la séparation des pouvoirs en protégeant les organismes fonctionnant sous une autorité législative contre des interventions inappropriées dans des matières qui leur auront été confiées par les législatures.

\section{B. Révision de la méthode pragmatique et fonctionnelle}

Dunsmuir a apporté un deuxième changement au droit du contrôle judiciaire. Il a révisé la méthode pragmatique et fonctionnelle qu'avait introduite l'arrêt Bibeault ${ }^{37}$ et que l'arrêt Pushpanathan avait précisée ${ }^{38}$. Les juges de la Cour se sont entendus pour reconnaître qu'un réexamen de la méthode pragmatique et fonctionnelle s'imposait. L'application de cette méthode exigeait beaucoup d'énergie et de capacité de divination des avocats lorsqu'ils essayaient de prédire le résultat de sa mise en œuvre dans une affaire donnée. Comme je l'avais affirmé dans mes motifs concurrents dans l'arrêt Chamberlain, l'application mécanique de cette méthode, telle une série de facteurs à vérifier l'un après l'autre, comme si elle faisait partie d'une sorte de liste, n'était ni pragmatique, ni fonctionnelle ${ }^{39}$.

Au moment de l'arrêt Dunsmuir, un membre de la Cour avait toutefois exprimé son scepticisme à l'égard du changement de nom d'une formule qui demeurait, à son avis, toujours essentiellement la même, malgré les modifications que proposait ce jugement dans le cadre de sa révision des méthodes de contrôle judiciaire ${ }^{40}$. Toutefois, la modification de la méthode pragmatique et fonctionnelle par la majorité de la Cour impliquait beaucoup plus qu'un changement de nom. L'approche proposée par la majorité part d'une perspective plus holistique. Elle demande à la cour de révision de décider si elle doit adopter une attitude de déférence à l'égard d'une ca-

Ibid. aux para. $48-49$

37 U.E.S., Local 298 c. Bibeault, [1988] 2 R.C.S. 1048, 95 N.R. 161 [Bibeault].

38 Pushpanathan c. Canada (Ministre de la Citoyenneté et de l'Immigration), [1998] 1 R.C.S. 982, 160 D.L.R. (4e) 193 [Pushpanathan].

39 Chamberlain c. Surrey School District No. 36, 2002 CSC 86, [2002] 4 R.C.S. 710 au para. 195, 221 D.L.R. (4e) 156 [Chamberlain].

40 Dunsmuir, supra note 1 au para. 121, juge Binnie. 
tégorie particulière de décisions administratives, par un examen de tous les facteurs pertinents vus dans leur ensemble.

La méthode de contrôle judiciaire comprend alors deux étapes. D'abord, la cour de révision détermine si une catégorie de décision a déjà été examinée par les tribunaux. Si la jurisprudence indique qu'une norme particulière s'applique à cet égard, le tribunal pourrait alors l'adopter sans effectuer une analyse complète. En effet, la jurisprudence peut comprendre plusieurs exemples de la norme appropriée pour des catégories particulières de questions. Ainsi, la norme de la décision raisonnable devrait être un choix à peu près automatique quand une cour examine des questions de fait, de politique ou de discrétion, dans les questions mixtes de fait et particulièrement celles où les faits et le droit sont difficiles à dissocier ${ }^{41}$. La présence d'une clause privative suggère aussi fortement que la norme du raisonnable s'applique, même si elle n'est pas déterminante ou décisive en soi $^{42}$. La jurisprudence existante confirme aussi l'existence de plusieurs cas où l'expertise d'un tribunal spécialisé justifiera l'application de la norme du raisonnable, même à l'égard de questions de droit. Cette application se justifie lorsque le tribunal interprète sa loi constitutive ou traite de questions de droit ou de législation, à l'égard desquelles il possède une expérience et des connaissances spécifiques ${ }^{43}$. Par contre, un certain nombre de questions de droit exigeraient l'application de la norme de la décision correcte. Comme je l'ai écrit dans Toronto c. S.C.F.P., certaines questions de droit de nature générale peuvent être d'importance centrale pour le système juridique dans son ensemble et se situer hors du domaine d'expertise d'un décideur spécialisé. De telles questions devraient être décidées correctement ${ }^{44}$. De même, une cour supérieure a le droit de substituer son opinion à celle du tribunal au sujet des questions constitutionnelles, dans le respect des fonctions dévolues aux cours supérieures en vertu des articles 96 à 101 de la Constitution ${ }^{45}$.

La majorité dans Dunsmuir souligne également que de véritables questions de compétence ou de constitutionnalité seraient généralement des matières à l'égard desquelles les cours supérieures devraient appliquer la norme de la décision correcte. Cependant, en reconnaissant l'existence de véritables questions de compétence, la Cour ne veut pas faire revivre les débats qui ont marqué la jurisprudence avant l'arrêt

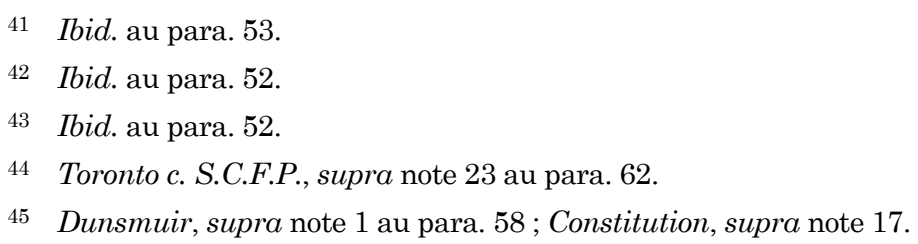


S.C.F.P. c. Société des alcools du Nouveau-Brunswick46, en 1979. Elle ne veut pas inviter les tribunaux à intervenir indûment dans les décisions des tribunaux et des corps administratifs. Dunsmuir s'attache plutôt à souligner l'importance pour les tribunaux administratifs de bien définir les limites des délégations d'autorité qui leur ont été faites. Les tribunaux administratifs doivent alors établir les paramètres de leurs actions correctement pour éviter les interventions des cours supérieures. Le même raisonnement s'appliquera à l'utilisation de la norme de la décision correcte au règlement des problèmes de conflits de juridiction entre des tribunaux spécialisés ${ }^{47}$. Cependant, ce type d'intervention exige une interprétation robuste de la compétence du décideur contrôlé. Il faut éviter de qualifier de question de compétence toutes les matières autres que la question étroite qui consiste à déterminer si une matière se situe bien à l'intérieur des pouvoirs délégués à un décideur administratif ${ }^{48}$.

En l'absence de jurisprudence susceptible d'orienter le tribunal, celuici doit s'engager dans la seconde étape de la méthode de contrôle. Dans ce cas, une cour doit effectuer une analyse contextuelle qui prend en compte plusieurs facteurs : l'existence d'une clause privative, l'objectif de la création de l'organisme, la nature de la question posée et l'expertise de l'organisme administratif. L'analyse de contrôle judiciaire doit éviter l'examen mécanique de chacun de ces facteurs. Chacun de ceux-ci n'est pas nécessairement pertinent dans un cas particulier. Je souligne que l'on doit les considérer de manière contextuelle, en donnant parfois préséance au facteur qui paraît le plus pertinent à une analyse particulière ${ }^{49}$. Dans une opinion minoritaire, la juge Deschamps a suggéré, à cet égard, qu'on devrait porter une attention particulière à la nature même de la question examinée ${ }^{50}$. La majorité de la Cour n'a cependant pas voulu établir une hiérarchie dans l'importance des facteurs à considérer. La norme de contrôle judiciaire demande à une cour d'adopter une attitude holistique qui prend en compte les principes fondamentaux sous-jacents à l'identification du choix d'une norme de contrôle, le maintien de la primauté du droit et le respect de la suprématie législative. Elle invite à aller au cœur de la matière et à éviter des débats indûment complexes sur la formulation de la norme de contrôle.

\footnotetext{
46 [1979] 2 R.C.S. 227, 97 D.L.R. (3e) 417 [S.C.F.P. avec renvois aux R.C.S.].

47 Dunsmuir, supra note 1 au para. 61.

48 Ibid. au para. 59. Voir aussi S.C.F.P., supra note 46 à la p. 233.

49 Dunsmuir, supra note 1 au para. 64.

$50 \quad$ Ibid. aux para. 158-66.
} 


\section{L'arrêt Khosa}

Dunsmuir traitait du contrôle judiciaire de common law. Il n'avait toutefois pas examiné le problème des rapports de celui-ci avec les régimes législatifs de contrôle judiciaire que l'on retrouverait dans des lois fédérales ou provinciales. L'importance de ce problème dépend largement de la nature des dispositions législatives en question. Par exemple, on retrouvait ce type de problème dans l'application de la Loi sur les Cours fédéra$l e s^{51}$, qui accorde une compétence générale à la Cour fédérale dans le contrôle de l'action des organismes administratifs fédéraux. La Cour suprême du Canada n'avait pas eu alors à examiner ses dispositions et notamment celles de son article 18.1. Celui-ci paraissait définir des cas particuliers dans lesquels la cour devrait ou non exercer son pouvoir de contrôle judiciaire ${ }^{52}$. Par ailleurs, en Colombie-Britannique, l'Administrative Tribunals Act prévoyait que le contrôle judiciaire relatif à certains sujets devait s'exercer suivant la norme du manifestement déraisonnable ${ }^{53}$. Cependant, la loi elle-même ne définissait pas la norme du manifestement raisonnable et s'en remettait à la common law sur ce sujet ${ }^{54}$.

Dans Khosa, la Cour suprême du Canada a été confrontée au problème des rapports entre le contrôle judiciaire prévu par la Loi sur les Cours fédérales et le contrôle judiciaire de common law. Notre Cour s'est alors prononcée sur l'articulation entre les dispositions de l'article 18.1 de la Loi sur les Cours fédérales et la méthode d'analyse prescrite dans l'arrêt Dunsmuir. En bref, l'opinion majoritaire a conclu que les dispositions de l'article 18.1 de la Loi sur les Cours fédérales n'écartaient pas la méthode d'analyse recommandée par l'arrêt Dunsmuir ${ }^{55}$. Des opinions concurrentes quant au résultat de l'appel, mais dissidentes sur ce point, estimaient plutôt que la loi écartait le contrôle judiciaire de common law et que la $L o i$ sur les Cours fédérales établissait non seulement des cas d'intervention, mais aussi des normes de contrôle ${ }^{56}$.

Le pourvoi portait sur une demande de contrôle judiciaire en vertu de l'article 18.1 de la Loi sur les Cours fédérales contre une décision d'un tribunal administratif, la Section d'appel de l'immigration de la Commission

L.R.C. 1985 , c. F-7.

Ibid., art. 18.1.

54 Pour un commentaire sur l'interaction entre cette loi et Dunsmuir, voir Mark Underhill, « Dunsmuir v. New Brunswick: A Rose by Any Other Name ... » (2008) 66 Advocate 343 à la p. 350 .

55 Khosa, supra note 2 aux para. 26,36 .

$56 \quad$ Ibid. au para. 69, juge Rothstein, au para. 138, juge Deschamps. 
de l'immigration et du statut de réfugié. En l'espèce, Khosa, un immigrant reçu très jeune en résidence permanente au Canada, avait été déclaré coupable de négligence criminelle causant la mort et condamné à une peine d'emprisonnement de deux ans moins un jour. En raison de cette condamnation, le gouvernement fédéral ordonna son renvoi dans son pays d'origine, l'Inde. Khosa forma un appel administratif devant la Section d'appel de l'immigration. Il demanda à celle-ci d'annuler le renvoi et d'autoriser la continuation de son séjour au Canada, en prenant des mesures spéciales pour des motifs d'ordre humanitaire en vertu de l'article 67 de la Loi sur l'immigration et la protection des réfugiés ${ }^{57}$.

La Section d'appel rejeta la demande et confirma l'ordre de renvoi. Khosa présenta alors une demande de contrôle judiciaire devant la Cour fédérale en vertu de l'article 18.1 de la Loi sur les Cours fédérales. Le juge de première instance conclut que la norme du manifestement déraisonnable gouvernait la décision de la Section et rejeta la demande de contrôle qui soulevait, selon lui, principalement des questions d'appréciation de la preuve. Khosa interjeta appel devant la Cour d'appel fédérale. La majorité de celle-ci conclut que la norme applicable était celle du raisonnable et que la Section d'appel avait effectué une analyse déraisonnable de la preuve. En conséquence, elle annula la décision attaquée.

L'affaire fut portée devant la Cour suprême du Canada par le ministre de la Citoyenneté et de l'Immigration. Une des questions principales débattues à l'occasion devant notre Cour fut celle de la nature du système de contrôle judiciaire applicable à l'examen de la décision de la Section d'appel. Selon certaines prétentions, l'article 18.1(4) de la Loi sur les Cours fédérales déterminait le cadre de l'intervention des cours fédérales. Il n'autorisait pas l'emploi de la méthode de common law, révisée par l'arrêt Dunsmuir. À l'inverse, une autre argumentation voulait que le recours à la méthode d'analyse pragmatique et fonctionnelle, comme l'avait modifiée l'arrêt Dunsmuir, demeurait pertinent. La Cour suprême du Canada se divisa à l'occasion de cet appel. Une majorité endossa l'opinion du juge Binnie, selon laquelle la Loi sur les Cours fédérales déterminait des cas d'intervention, mais ne définissait pas des normes de contrôle. Selon cette opinion, la Loi sur les Cours fédérales n'excluait pas la méthode d'analyse de l'arrêt Dunsmuir. La majorité décida que la norme du raisonnable s'appliquait et confirma la décision de la Section d'appel. Deux juges, les juges Rothstein et Deschamps, conclurent que la codification du régime de contrôle judiciaire dans la Loi sur les Cours fédérales écartait le régime de common law. Cependant, ils reconnurent la validité de la décision de la Section d'appel, puisque Khosa n'avait pas établi l'existence de

57 L.C. 2001 , c. 27. 
l'un des cas d'intervention judiciaire prévus par la Loi sur les Cours fédérales.

L'arrêt Khosa confirme la validité de l'approche adoptée dans Dunsmuir. Il retient l'existence de deux normes de contrôle. Il reconnaît la validité et la pertinence de la méthode d'analyse prescrite dans cet arrêt, en présence de règles législatives comme celles qu'édicte la Loi sur les Cours fédérales. Surtout, il réaffirme l'importance du concept de déférence dont la pertinence ne dépend pas seulement de la présence d'une clause privative. L'arrêt Khosa admet que dans les cas appropriés, la cour de révision s'incline devant les choix interprétatifs de certaines règles de droit, l'évaluation de la preuve et les solutions adoptées par le décideur soumis au contrôle judiciaire ${ }^{58}$. Cette solution ne rejette pas complètement la légitimité du pluralisme juridique dans les limites acceptées par l'arrêt Dunsmuir. L'arrêt écarte ainsi l'approche prônée par les juges Rothstein et Deschamps, qui aurait imposé un contrôle d'exactitude à l'égard de toutes les questions de droit en l'absence d'une clause privative suffisamment explicite et forte.

\section{Conclusion}

Khosa marque ainsi une étape importante dans le développement du cadre juridique du contrôle judiciaire à la suite de l'arrêt Dunsmuir. Toutefois, je le qualifie toujours d'étape, puisque la longue histoire du contrôle judiciaire et de l'évolution de cette partie du droit administratif n'est sans doute pas terminée. Les arrêts Dunsmuir et Khosa sont des moments d'une recherche d'un système de contrôle judiciaire plus simple et plus efficace, destiné à assurer non seulement l'efficacité des procédures administratives, mais également le respect des principes fondamentaux de l'ordre juridique et constitutionnel canadien. Quel sera le terme de cette route? Je l'ignore. Le temps et notre expérience nous le diront. Vous aurez, sans aucun doute, l'occasion de contribuer largement au déroulement de cette histoire.

58 Khosa, supra note 2 aux para. 25-26. 